\title{
19 Rektumprolaps
}

Reinhard Ruppert

\subsection{Kapitelzusammenfassung}

In diesem Kapitel wird das Problem des kompletten äußeren Rektumprolaps mit seinen Unterformen ursächlich dargestellt. Besonders wichtig bei dieser funktionellen Störung sind die zahlreichen Möglichkeiten der Therapie. Die richtige Indikationsstellung und die Wahl des entsprechenden therapeutischen Verfahrens sollen hier ausführlich dargestellt werden.

\subsection{Definition}

Das Rektum schließt direkt an den Analkanal an und dient als Vorratsbehälter. Die unteren $2 / 3$ des Mastdarms sind eingebettet in das mesorektale Fett und Lymphknotenpaket, beides liegt somit extraperitoneal. Das Mesorektum ist dorsalseitig locker vor der Waldeyerschen Faszie fixiert. Zwischen Faszie visceralis und parietalis zeigt sich ein lockerer, verschieblicher Spalt. Lediglich das obere Drittel des Rektums liegt intraperitoneal und ist relativ frei beweglich. Die ventrale Fixation des Rektums ist hier lediglich zur Scheide hin und damit deutlich mobiler. Kommt es nun im Rahmen von Veränderungen (Geburtstrauma, erhöhter Elastingehalt des Gewebes usw.) zu einer Auflockerung, dann kann sich das Rektum zunächst vor allem ventral und dann in der Folge auch dorsal soweit lockern, dass es zu einer Vollwandausstülpung kommt.

\section{3 Ätiologie, Pathogenese, Inzidenz}

Der Rektumprolaps ist keine selbstständige Erkrankung, sondern eine funktionelle Störung mit zugrundeliegenden multiplen Ursachen. Die ursprünglich von Moscowitz 1912 zugrunde gelegte Theorie einer Gleithernie kann heute als widerlegt betrachtet werden. Multiple Faktoren spielen hier eine Rolle. Genetisch bedingte Unterschiede im Aufbau der Haut bzw. in der Zusammensetzung des Elastinanteils können eine Rolle spielen. Häufige anatomische Veränderungen wie tiefer Douglas, aufgelockerter, diastatischer M. levator ani, als auch laxe fasziale Aufhängungen finden sich im Zusammenhang mit dem Rektumprolaps. Am häufigsten betroffen sind ältere Frauen gegenüber Männern in einem Verhältnis von 10:1. Die Inzidenz beträgt 2,5 pro 100.000 Personen.

Im Kindesalter tritt diese Veränderung nur sehr selten auf. Wenn, dann sind Kinder im Alter unter 3 Jahren betroffen. Hier spielen andere Faktoren wie Mangelernährung oder chronisch respiratorische Erkrankungen wie zystische Fibrose eine Rolle. 


\subsection{Symptomatik}

Die häufigsten Symptome des Rektumprolaps sind äußerst unspektakulär. Nässen, bluten, jucken und schmieren sind die erstgenannten Beschwerden. Stuhlinkontinenz und fraktionierte Entleerung werden dann erst in der Folge berichtet. Über das prolabieren des gesamten Rektums berichten die wenigsten Patienten.

\subsection{Klassifikation}

Es lassen sich grundsätzlich zwei Formen unterscheiden:

1. kompletter äußerer (externer) Rektumprolaps (auch manifester Rektumprolaps)

2. innerer (interner) Rektumprolaps (IRP) - Intussuszeption

Der IRP wiederum lässt sich in drei weitere Untergruppen aufteilen:

a. Grad I - Prolaps bis oberhalb des M. puborektalis (PRS)

b. Grad II - Prolaps bis auf Höhe des M. puborektalis

c. Grad III - Prolaps bis in den Analkanal, aber nicht über den Analrand hinaus.

Andere Einteilungen sind beschrieben, unterschieden sich aber nur unwesentlich.

\subsection{Diagnostik und Differentialdiagnosen}

Die klinische Untersuchung steht hier im Vordergrund. Eine Untersuchung in Linksseitenlage oder in Steinschnittlage zeigt bereits inspektorisch die Veränderungen. Durch das Schmieren und Nässen findet sich häufig ein perianales Kontaktekzem. Bei Entspannung zeigt sich der After klaffend (Internusrelaxation). Die digitale Untersuchung vermittelt meist sofort den Eindruck eines schlaffen, widerstandslosen Afters. Ein forciertes Valsalvamanöver zeigt sofort das komplette Ausstülpen des Rektums (Abb.19.1). Ist die Veränderung noch im Stadium des IRP, dann kann im Proktoskop das Ausmaß der Intussuszeption (Höhenlokalisation zum PRS) beurteilt werden. Die Rektoskopie kann Veränderungen im Sinne eines Ulcus recti an der Vorderwand zeigen. Dieses ist immer ein indirektes Zeichen für einen IRP oder einem kompletten Rektumprolaps insbesondere bei Männern. Sollte die unphysiologische Lagerung (Linksseite, Steinschnittlage) nicht ausreichend zur klinischen Diagnostik sein, kann die Untersuchung auf einem sog. Spiegelstuhl in natürlicher Defäkationshaltung durchgeführt werden (Abb. 19.2).

Bei einem kompletten äußeren Rektumprolaps sind keine weiteren bildgebenden Verfahren mehr notwendig. Sind die Veränderungen aber im Sinne eines IRP, dann sollte ein dynamisches MRT Defäkographie zur weiteren Beurteilung durchgeführt werden. Die konventionelle Defäkographie ist aus strahlenschutzrechtlichen Gründen nicht mehr indiziert. 

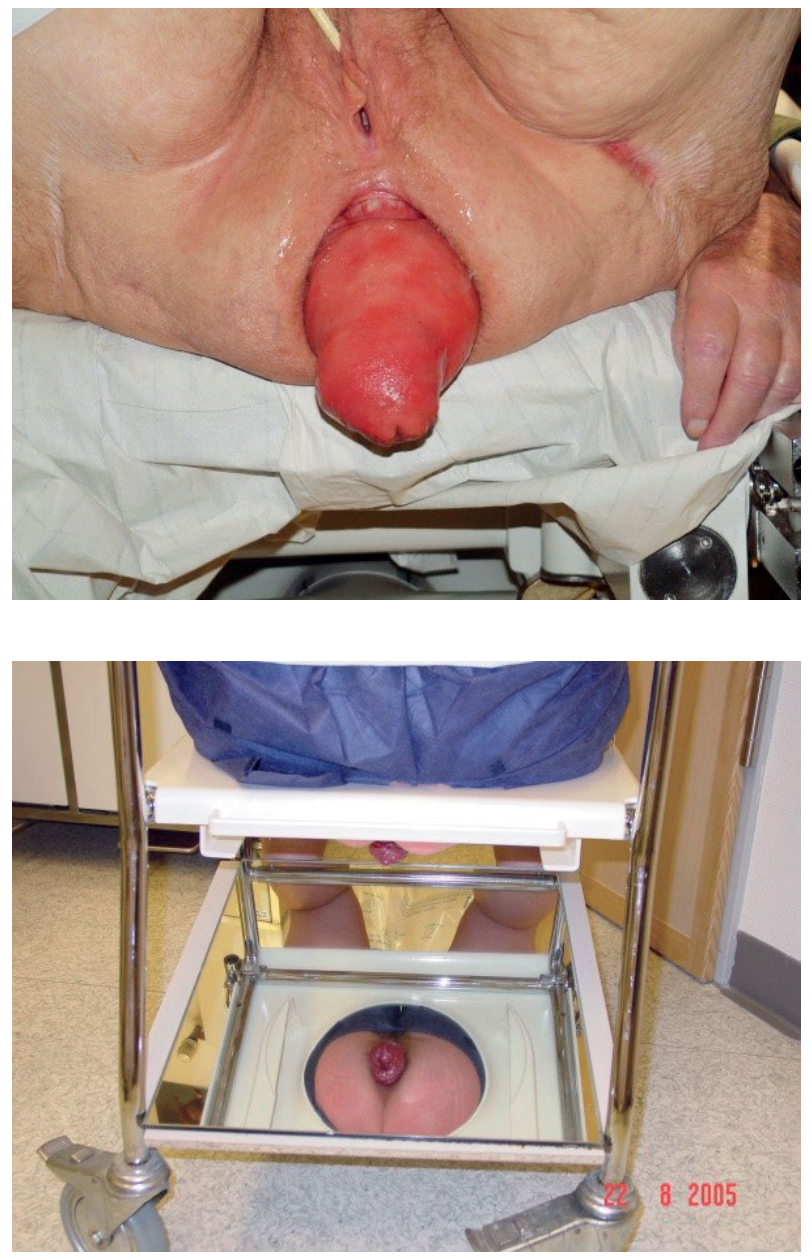

Abb.19.1: Kompletter Rektumprolaps.

Abb. 19.2: Rektumprolaps im Spiegelstuhl.

Manometrie und Nervenleitgeschwindigkeitsmessungen (Pudendus) sind ohne klinische Relevanz und sollten nicht mehr durchgeführt werden. Die Kolontransitzeitbestimmung kann bei anamnestischer ausgeprägter Obstipation bzw. Darmträgheit sinnvoll sein.

Differentialdiagnostisch ist es wichtig einen ausgeprägten Analmukosaprolaps $\mathrm{zu}$ unterscheiden. Beim Ulcus recti des Mannes ist vor allem die Unterscheidung zum funktionellen „Presser“ wichtig. Hier muss ein gestörtes Defäkationsverhalten ausgeschlossen werden. Psychische und psychosomatische Störungen sind auszuschließen.

Komplexe Beckenbodenstörungen mit Genitalprolaps und Harninkontinenz sind fachübergreifend mit Gynäkologe und Urologe zu beurteilen. 


\subsection{Indikation}

Maßgebend für dieses funktionelle und benigne Leiden ist der Leidensdruck. Abhängig vom Ausmaß der Einschränkung der Lebensqualität ist die Indikation zur Operation gegeben. Es handelt sich nicht um eine lebensbedrohliche Erkrankung. Die Stadien des IRP sind sehr differenziert zu betrachten. Konservative Maßnahmen können hier noch ausreichend sein. Beim alleinigen kompletten Rektumprolaps oder in Kombination mit weiteren Beckenbodenstörungen wie Vaginalprolaps, Genitalprolaps und dadurch bedingter dauerhafter Schädigung des Sphinkters ist die chirurgische Versorgung indiziert.

\subsection{Konservative Therapie}

Die konservative Therapie ist sehr eingeschränkt möglich. Sie umfasst vor allem die Stuhlregulation und die Komplettierung der Entleerung. Bei einer Intussuszeption niederen Grades mit Rektozele kann eine Pessareinlage funktionieren. Die Inkontinenzproblematik bei abgeschwächtem Sphinkter lässt sich nur partiell mit Analtampon beim IRP kompensieren. Die konservative Therapie dient somit in erster Linie der Therapie der Begleitveränderung.

\subsection{Operative Therapie}

In der Literatur sind über 100 verschiedene Operationsverfahren bekannt. Dies ist ein Hinweiss darauf, dass das perfekte Verfahren noch nicht gefunden ist. Grundsätzlich läßt sich zwischen perinealen Verfahren und transabdominellen Verfahren unterscheiden. Das häufig fortgeschrittene Alter der Betroffenen stellt heute keine Kontraindikation mehr dar. Moderne Anästhesieverfahren mit niedrigem Operationsrisiko erlauben heute immer einen chirurgischen Therpieansatz. Wichtig ist das Ausmaß des Vorfalls und die Lebensqualitätseinschränkung.

\subsubsection{Perineale Operationsverfahren}

Die häufigsten perinealen Verfahren sind die Operation nach Rehn-Delorme und die Altemeier-Resektion. Vorteile dieser Verfahren sind die Unversehrtheit der Abdominalwand und die Möglichkeit, diese Verfahren in Leitungsanästhesie durchzuführen. Hintergrund war letztendlich ein Verfahren anzubieten, das ein vermutlich niedrigeres Narkoserisiko ermöglicht. In Zeiten moderner, schonender Anästhesieverfahren ist dies sicherlich hinfällig. Nachteil der perinealen Verfahren ist die eingeschränkte 


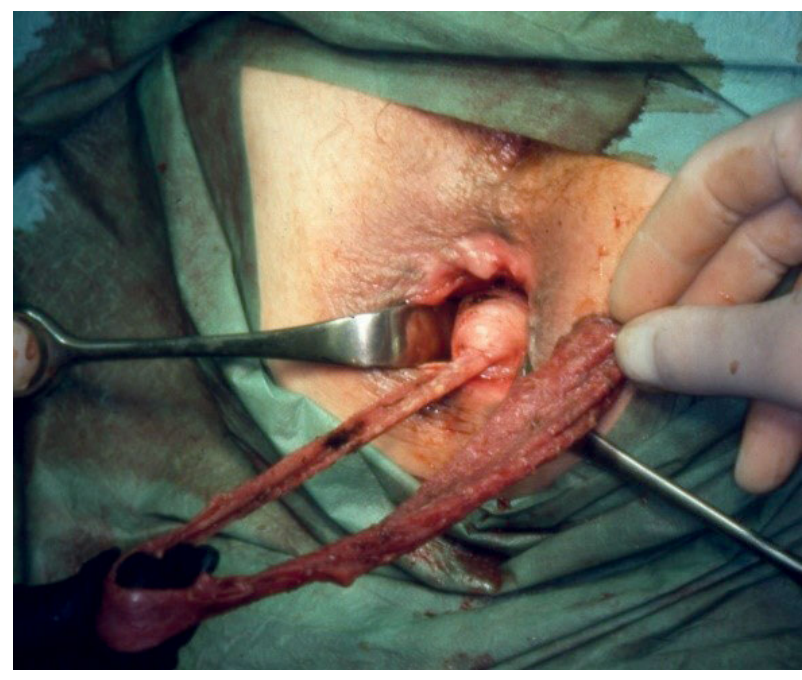

Abb. 19.3: Rehn DelormeMukosazylinder.

Korrekturmöglichkeit simultan bestehender Beckenbodenpathologien (Scheidenblindsackvorfall, Genitalprolaps).

\section{Rehn-Delorme}

Dieses Verfahren eignet sich vor allem für kleinere Prolapsformen ohne Begleitpathologien. Nach vollständiger Luxation des Prolaps, wird ein cirkulärer Mukosazylinder präpariert und anschließend die Rektumwand wie eine Ziehharmonika an 4 Fixpunkten (12, 3, 6 und 9 Uhr SSL) gerafft. Die Komplikationsrate ist sehr niedrig (Abb. 19.3).

\section{Altemeier Resektion}

Bei diesem Verfahren wird nach kompletter Luxation des Prolapses die Rektumvorderwand, $1 \mathrm{~cm}$ distal der linea dendata, der Douglasraum eröffnet. Im weiteren wird dann schrittweise die Rektumwand komplett durchtrennt. Das Rektum zusammen mit dem Sigma herusgezogen und darmwandnah skelettiert. Die Präperation erfolgt soweit, bis kein Kolon mehr luxiert werden kann. Der Darmanteil wird reseziert und anschließend eine koloanale Anastomose in End zu End oder Seit zu End Technik angelegt. Die Anastomosierung kann sowohl als Handnaht als auch als Stapleranastomose durchgeführt werden. Funktionell kommt es hier zu einem kompletten Verlust des Rektums als Reservoir im Sinne einer tiefen Rektumresektion mit entsprechenden funktionellen Veränderungen im Sinne eines LARS Syndroms (low anterior rectal resection syndrom). 


\section{Modifizierter Stapler Altemeier - Perineale Stapler Prolapsresektion (PSPR)}

Dieses etwas vereinfachte Verfahren ist für Prolapsformen bis $10 \mathrm{~cm}$ geeignet. Hier wird der präanal hervorluxierte Prolaps mit einem Linearcutter bei 3, 6, 9 und $12 \mathrm{Uhr}$ ca. $1 \mathrm{~cm}$ distal der linea dendata axial durchtrennt (Abb. 19.4). Die Rektumhinter- und Vorderwand werden dann in einem weiteren Schritt entweder mit einem Linearcutter oder semizirkulären Stapler durchtrennt (Abb. 19.5). Die Klammernähte werden dann mit einer fortlaufenden, durchgreifenden Naht übernäht. Nahtinsuffizienzen oder Verletzungen des Dünndarmes sind möglich. Die in der Literatur angegeben Rezidivraten liegen bei ca. $20 \%$ und höher.
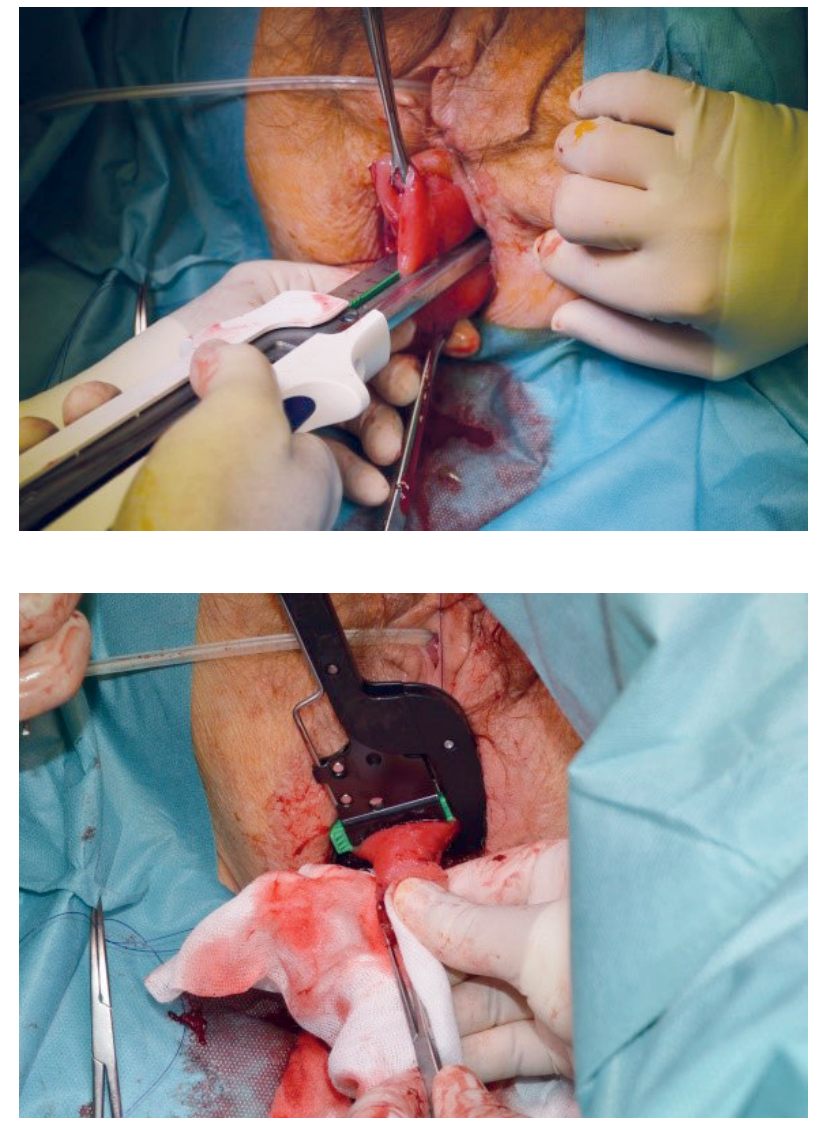

Abb. 19.4: Linearstapler 3:00 Uhr.

Abb. 19.5: Absetzen mit dem Linearcutter. $35 \mathrm{~mm}$ quer. 


\subsubsection{Transabdominelle Verfahren}

Transabdominelle Verfahren eignen sich vor allem für die Behandlung mehrerer Pathologien in den verschiedenen Kompartimenten in einer Operation. Hierbei wird durch die komplette dorsale und laterale Mobilisation des Rektums bis in den Analkanal hinein das dorsale Kompartiment stark eleviert und auf Höhe des Promontoriums und auf Höhe S2 fixiert. Simultan zur Anhebung dorsal wird bei erhaltener ventraler Fixierung (Douglas) gleichzeitig das mittlere Kompartiment mit Vagina und Uterus ebenfalls angehoben. Die Fixierung kann durch einfache Nahtrektopexie (Sudeck 1922/Loygue 1983) oder durch ein Kunststoffnetz erfolgen. Die Netzoperationen nach Ripstein und Wells sind allerdings in den letzten Jahren aus der Mode gekommen. Eine weitere Möglichkeit ist die ventrale Rektopexie mit Faszienstreifen wie sie ursprünglich von Orr 1947 erstmals beschrieben wurde. Die Methode wurde dann in Frankreich durch Loygue 1983 weiterverbreitet und wurde zu einer der alternativen Operationsmethoden. Aber über Frankreich hinaus konnte sich diese nicht etablieren. Erst durch A. D’Hoore im Jahre 2004 erlebte die Methode eine rasante Verbreitung. Dieses Verfahren wird in der Regel laparoskopisch durchgeführt. Das Verfahren verzichtet auf die komplette dorsale Mobilisation des Rektums. Hiermit können die Nerven optimal geschont werden. Auf Höhe des Promontoriums wird in der Regel rechtseitig das Beckenperitoneum inzidiert bis ins kleine Becken und ventral semicirculär durch das Spatium rektovaginale geführt bis zur Gegenseite. Präparation des Spatium rektovaginale bis zu seinem tiefsten Punkt für die spätere Fixation des Netzes. Nach Hysterektomie ist ein vaginal eingeführter Manipulator (Phantom) hilfreich, um den Einstieg in das Spatium zu erleichtern. Eine Eröffnung des Vaginalstumpfes sollte vermieden werden. Anschließend erfolgt die Einlage eines konfektionierten nicht resorbierbaren Netzes, das ventral am tiefsten Punkt des Spatium rektovaginale am Rektum fixiert wird. Danach erfolgt die Fixation an der Rektumvorderwand mit simultaner Fixation der Vaginahinterwand mit und ohne Uterus. Das Band wird dann schräg über das Rektum zum Promontorium geführt, wo die abschließende Fixierung erfolgt. Das Netz wird spannungsfrei eingebracht. Danach Verschluss des Peritoneums mit fortlaufender Naht.

Die klassische dorsale Pexie mit der Nahtfixation nach Sudeck kombiniert mit Sigmaresektion (Frykman/Goldberg) ist weiterhin eine optimale Alternative zur ventralen Pexie. Hier erfolgt die komplette dorsale Mobilisation bis in den Analkanal hinein (Abb.19.6). Semicirculäre dorsolaterale Mobilisation mit Durchtrennen der lateralen Aufhängung, aber unter Erhalt der ventralen (Douglas) Fixierung. Legen von 2 Pexienähten nicht resorbierbares Fadenmaterial (Prolene) durch das Kreuzbein auf Höhe des Promontoriums (Abb.19.7). Durch Anhebung des Rektums (hinteres Kompartiment) wird das mittlere Kompartiment bei Erhalt der ventralen Fixierung (Mesorektum) mitgenommen, sodass hier ebenfalls eine Korrektur erfolgt (Abb.19.9). Wird die Nahtfixation durch das gesamte Mesorektum durchgeführt (Abb. 19.8), kann 


\section{$8-19$ Rektumprolaps}

eine optimale präsakrale Fixation erfolgen. Abschließend ebenfalls Verschluss des Peritoneums.

Dorsale Rektopexie nach Sudeck

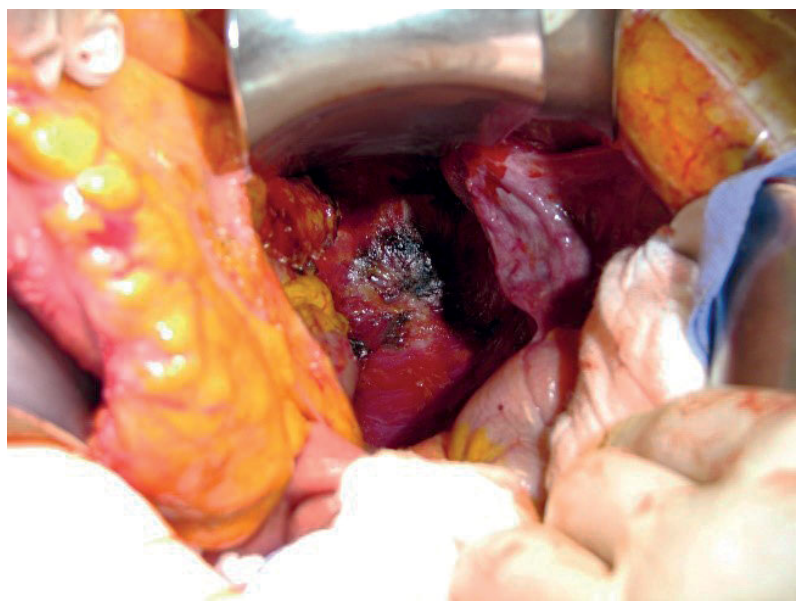

Abb. 19.6: Blick ins Becken.

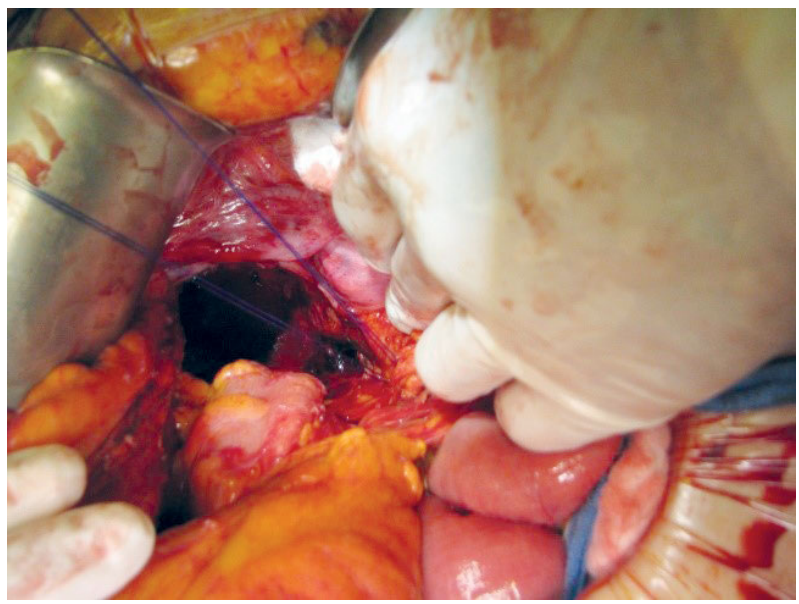

Abb. 19.7: Präsakrale Fixationsnaht. 


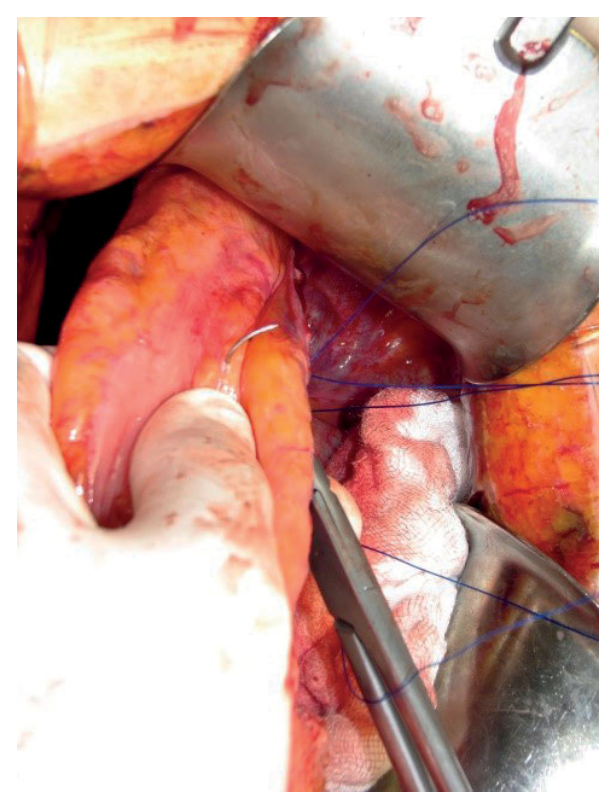

Abb. 19.8: Transmesorektale Fixierungsnaht.

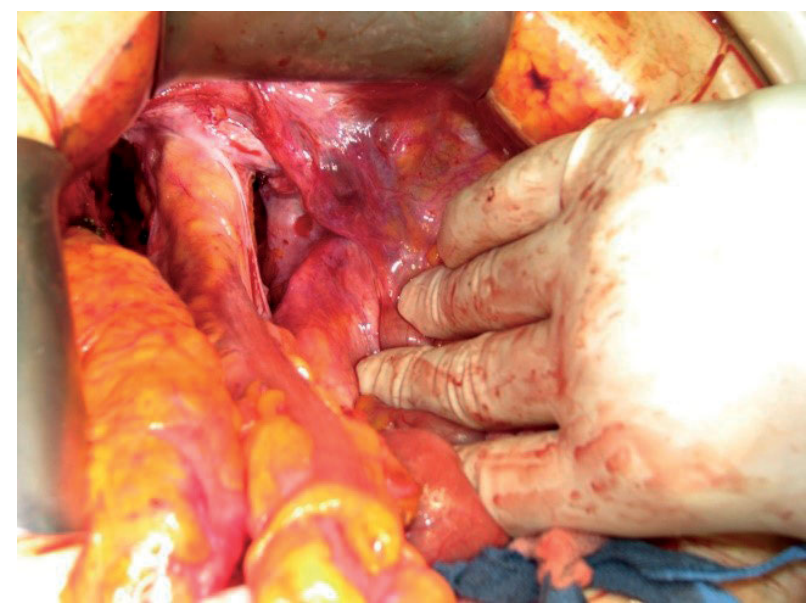

Abb. 19.9: Fixiertes Rektum.

\subsubsection{Komplikationen}

Bei allen genannten operativen Verfahren kann es zu Blutungen kommen. Arterielle Blutungen sind gut darstellbar und zu erkennen. Problematisch sind eher venöse Blutungen, die unter Umständen dann zu größeren, massiven Hämatomen insbesondere im Präsakralraum führen können. Bei den Resektionsverfahren ist die Nahtinsuffizienz im Rahmen der gängigen Kolonresektionen mit um die 5\% zu sehen. Bei den Netzassoziierten Verfahren ist das Problem der Netzarrosion sowohl ins Rektum als 
auch in die Vagina nicht zu vernachlässigen. Die Angaben in der Literatur schwanken zwischen 2 bis 4,6\%. Die gesamten Komplikationsraten schwanken ebenfalls zwischen 4 bis $46 \%$. Rezidivraten werden mit bis zu $15 \%$ angegeben.

Die klassischen Nahtrektopexieverfahren haben eine Rezidivrate von im Mittel $3 \%$ (0 bis 27\%). Außer den oben genannten allgemeinen Problemen gibt es keine Material assoziierten Komplikationen.

\subsubsection{Langzeitergebnisse}

Wirklich verlässliche Langzeitergebnisse der verschiedenen Verfahren im Vergleich gibt es nicht. Die 2013 veröffentlichte PROSPER Studie war die erste Arbeit, die versuchte, perineale Verfahren (Altemeier und Delorme) mit den transabdominellen Rektopexieverfahren prospektiv zu vergleichen. Aufgrund der zu geringen Fallzahl war die Studie underpowered und daher in ihrer Aussagekraft eingeschränkt. Daher kam es zu keinen signifikanten Unterschieden. Eines der wesentlichen Ergebnisse ist, dass die Lebensqualität unabhängig vom Verfahren positiv beeinflusst wird. Die Rezidivraten unterscheiden sich in der genannten Studie nicht signifikant voneinander (Altemeier 24\%, Delorme $31 \%$, Resektionsrektopexie 13\%, Sudeck 26\%). Die Ergebnisse der deutschen Multicenterstudie DELORES liegen aktuell noch nicht vor. Die Rekrutierung ist abgeschlossen, sodass jetzt die Nachbeobachtung komplettiert werden muss; diese endet 2021. Erste Zwischenergebnisse sind vielleicht $2019 \mathrm{zu}$ erwarten.

\subsection{Prävention}

Allgemeine Empfehlungen zur Prävention eines kompletten äußeren Rektumprolaps gibt es nicht. Neben den sicherlich genetisch bedingten Ursachen (erhöhter Elastingehalt der Haut) sind geburtstraumatisch bedingte Läsionen nur schwer zu vermeiden. Entbindungen mittels Sectio sind hier sicherlich präventiv zu betrachten. Eine allgemeine Empfehlung kann jedoch in dieser Hinsicht nicht ausgesprochen werden.

\section{Weiterführende Literatur}

Consten EC, et al. Long term outcome after laparoscopic ventral mesh rectopexy: an observational stud of 919 consecutive patients. Ann.Surg. 2015;262(5):742-747.

Davilla GW, Ghoniem GM, Nasseri Y. Pelvic Floor Dysfunction 2006 ; Springer Verlag, ISBN 1-85233730-3.

D`Hoore et al. Long - term outcome of laparoscopic ventral rectopexy for total rectal prolapse. British journal of Surgery. 2004;91:2000-2005.

Eung JS. Surgical treatment of rectal prolapse. Journal of Korrean Society of Coloproctology. 2011;27(1):5-12. 
Portier G, et al. Surger for rectal prolapse: Orr - Logue, ventral rectopexy with limited dissection prevents postoperative induced constipation without increasing recurrence. Dis. colon Rectum. 2006;49:1136-1140.

Richelle JF, et al. Haemorrhoids, rectal prolapse, anal fissure, peri-anal fistulae and sexuall transmitted diseases. Best practice \& research clinical Gastroenterology. 23;2009:575-592.

Senapati A, et al. PROSPER a randomized comparison of surgical treatments for rectal prolapse. Colorectal Dis. 2013;15:858-870.

Solomon M, et al. Randomized clinical trial of laparoscopic versus open abdominal rectopexy for rectal prolapse. British Journal of Surger. 2002;89:35-39.

Sudeck P. Rektumprolaps Operation. Zentralblatt Chir. 1922;49:689. 
